\title{
Application of the digital image correlation method in the study of cohesive coarse soil deformations
}

\author{
Janusz P. Kogut ${ }^{1, *}$, and Marcin Tekieli ${ }^{1}$ \\ ${ }^{1}$ Cracow University of Technology, Faculty of Civil Engineering, Kraków, Poland
}

\begin{abstract}
Non-contact video measurement methods are used to extend the capabilities of standard measurement systems, based on strain gauges or accelerometers. In most cases, they are able to provide more accurate information about the material or construction being tested than traditional sensors, while maintaining a high resolution and measurement stability. With the use of optical methods, it is possible to generate a full field of displacement on the surface of the test sample. The displacement value is the basic (primary) value determined using optical methods, and it is possible to determine the size of the derivative in the form of a sample deformation. This paper presents the application of a non-contact optical method to investigate the deformation of coarse soil material. For this type of soil, it is particularly difficult to obtain basic strength parameters. The use of a non-contact optical method, followed by a digital image correlation (DIC) study of the sample obtained during the tests, effectively completes the description of the behaviour of this type of material.
\end{abstract}

Keywords: coarse soil, image correlation, unconfined compression

\section{Introduction}

It is difficult to identify the parameters to accurately describe soil. As is already known, soil is a mixture of solids, water, and gases. There are several methods to test soil. Namely, shear box tests and vane tests, as well as uniaxial and triaxial stress tests give some soil parameters, in a limited range, for different types of soils. In particular, they have caused problems for coarse and very coarse soils. Furthermore, it is difficult to extrapolate typical continuum models from coarse soils. Even after laboratory tests, it is necessary to implement the data into large, numerical scale models and then form a decision on whether the results are realistic, at least for a certain range of deformations (elastic, plastic). The last few decades have provided us with a tremendous opportunity to apply another class of the measurement methods, known as non-destructive tests. The internal structure of a soil sample may be examined for volumetric inspection with penetrating radiation (RT), such as

\footnotetext{
${ }^{*}$ Corresponding author: jkogut@pk.edu.pl
} 
X-rays, neutrons, or gamma radiation. A combination of such tests, with the method applied in this paper, appears to be promising. Digital image correlation (DIC) generally refers to another class of non-contacting methods that acquire images of an object in digital form and perform image analysis to extract sensor-plane motions that can then be converted into full-field measurements on the corresponding object. One of the most commonly used approaches employs random patterns and compares subsets from initial, reference configuration and then the next configuration images to obtain a full-field of sensor-plane measurements. Fig. 1 presents the idea of DIC method [1].
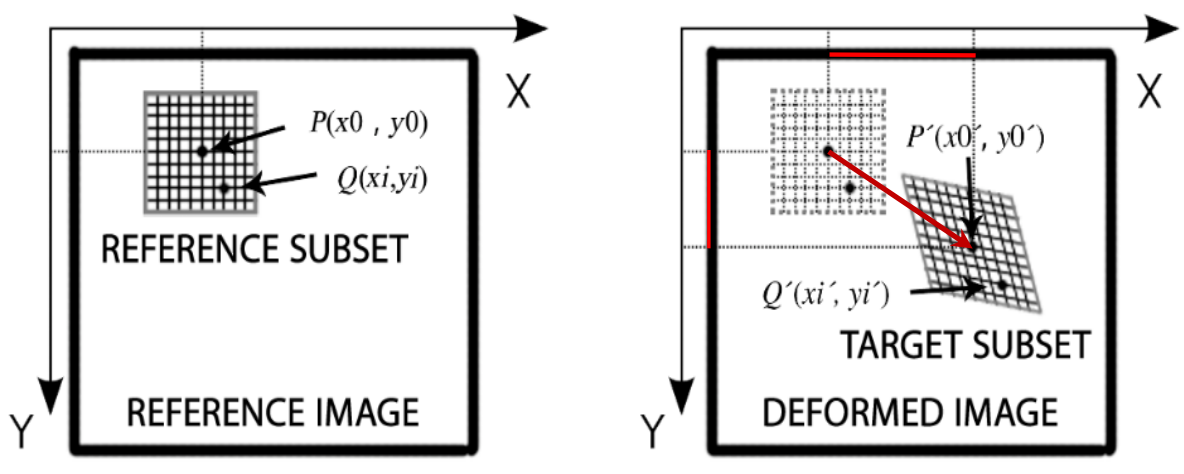

Fig. 1. The idea of DIC method - the reference image subset compared to the deformed target subset of points in the image processing [1].

The DIC method looks for the maximum of the correlation array between pixel intensity array subsets on several corresponding images, which gives the integer translational shift between them. It also estimates shifts in a finer resolution than the resolution of the original images, based on "subpixel" registration, because the measured shift is smaller than an integer pixel unit. An iterative approach may be used to maximize the interpolated correlation coefficient by using nonlinear optimization techniques. The two-dimensional discrete cross correlation $r_{i j}$ is defined here [2-3] as

$$
r_{i j}=\frac{\sum m \sum n\left[f_{(m+i, n+j)}-f\right]\left[g_{(m, n)}-\bar{g}\right]}{\sqrt{\sum m \sum n\left[f_{(m, n)}-\bar{f}\right]^{2} \sum m \sum n\left[g_{(m, n)}-\bar{g}\right]^{2}}}
$$

where: $f_{(m, n)}$ is the pixel intensity or the gray-scale value at a point $(m, n)$ in the original image, $g_{(m, n)}$ is the gray-scale value at a point $(m, n)$ in the translated image, $\bar{f}$ and $\bar{g}$ are mean values of the intensity matrices $f$ and $g$, respectively. In practical applications, one may use digital Fourier transformation (DFT) and then, by taking the complex conjugate and inverse Fourier transformation, receive the integer shift of $(\Delta x, \Delta y)=\arg _{(i, j)} \max \{r\}$. As for the deformation mapping, the mapping function that relates the images is derived by comparing a set of sub-window pairs over the whole images (fig. 1). The coordinates (grid points) namely $\left(x_{i}, y_{j}\right)$ and $\left(x_{i}{ }^{*}, y_{j}{ }^{*}\right)$ are related by the translations that occur between the two images. If the deformation is small and perpendicular to the optical axis of the camera, then the relation between $\left(x_{i}, y_{j}\right)$ and $\left(x_{i}{ }^{*}, y_{j}{ }^{*}\right)$ can be approximated by a $2 \mathrm{D}$ affine transformation such as: 


$$
\begin{aligned}
& x^{*}=x+u+\frac{\partial u}{\partial x} \Delta x+\frac{\partial u}{\partial y} \Delta y \\
& y^{*}=y+v+\frac{\partial v}{\partial x} \Delta x+\frac{\partial v}{\partial y} \Delta y
\end{aligned}
$$

where: $u$ and $v$ are translations of the centre of the subset in the $X$ and $Y$ directions, respectively. The distances from the centre of the subset to the point $(x, y)$ are denoted by $\Delta x$ and $\Delta y$. Thus, the correlation coefficient $r_{i j}$ is a function of displacement components $(u$, v) and relevant displacement gradients [4].

\section{Uniaxial unconfined compression soil tests}

Three soil samples have been tested in order to get the Young's modulus E. The soil material is a quaternary colluvium formation deposit of the Carpathian flysch [5]. The uniaxial unconfined compression tests of the soil samples have been performed. Fig. 2 presents the experimental setup. The experiment has been performed with the application of Zwick 1455 static testing machine. It has a maximum load range of up to $20 \mathrm{kN}$, while the force measurement resolution is equal to $0.1 \mathrm{~N}$, and the resolution of the vertical deformations measurements is equal to $0.002 \mathrm{~mm}$ [6-7]. The compression force and the vertical displacement has been measured. The camera used in the experiment is a Nikon DSLR D5300 (24Mpx, 6000x4000 px) with a Sigma 17-50 mm F/2.8 EX DC OS HSM lens.
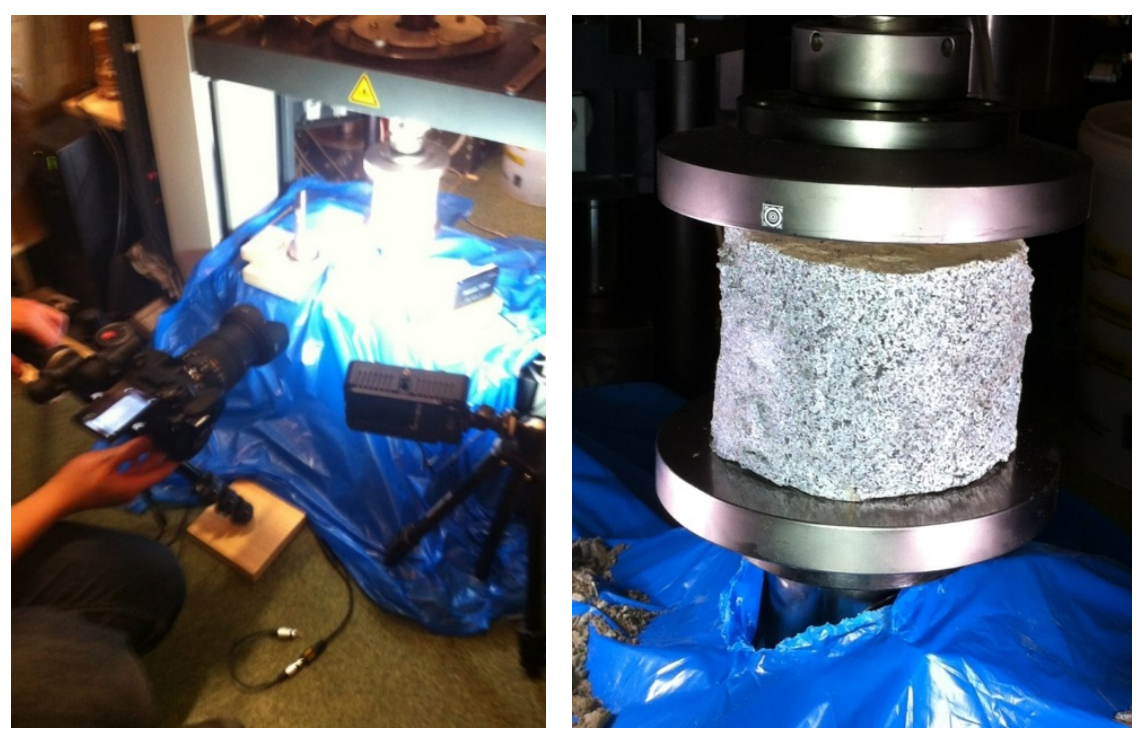

Fig. 2. The experimental setup consisting of the high resolution camera, Zwick 1455 static testing machine (left), and the soil sample tested (right).

The resolution refers to the original value, which is obtained by means of an optical measurement, and, in this case, it is the displacement of a given point (marker). Thanks to the use of sub-pixel measurement, the measurement resolution was, depending on the test, from 0.01 to $0.02 \mathrm{px}$, yielding 0.0004 and $0.0008 \mathrm{~mm}$, respectively. The collected image data has been processed using an optical measurement system (CivEng Vision) developed by the one of the authors of this paper [1,8-9]. 


\section{Discussion of results}

Fig. 3 presents the results of the deformations of the coarse soil sample in the solid state, in the following four virtual steps. It also may be visible, from the figure, that the virtual strain gauges were applied. The behaviour of the soil sample resembles the barrel shape with the vertical loading increasing, which may be seen when analysing steps \#1 to \#4. On the other hand, the sample cracks are noticeable at the bottom of left figure.
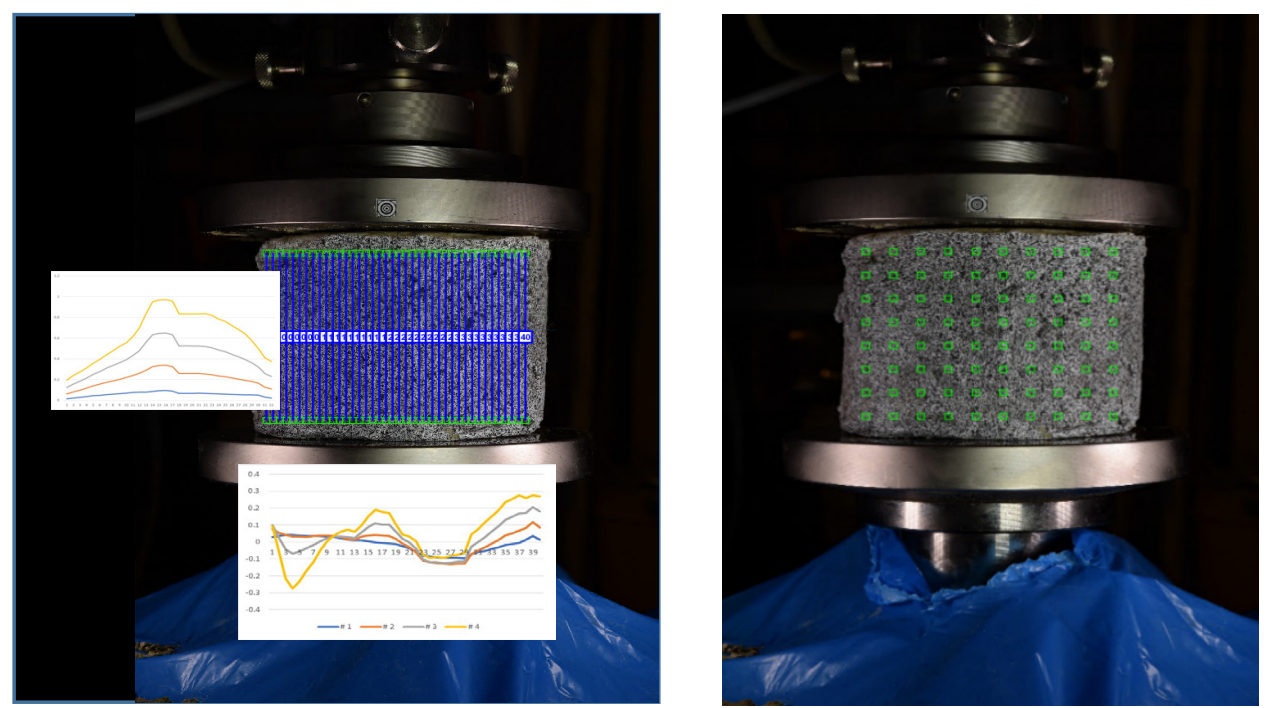

Fig. 3 The results of soil deformations in the four following steps of the compression test and the virtual strain gauges applied (left), and the virtual mesh grid (right).

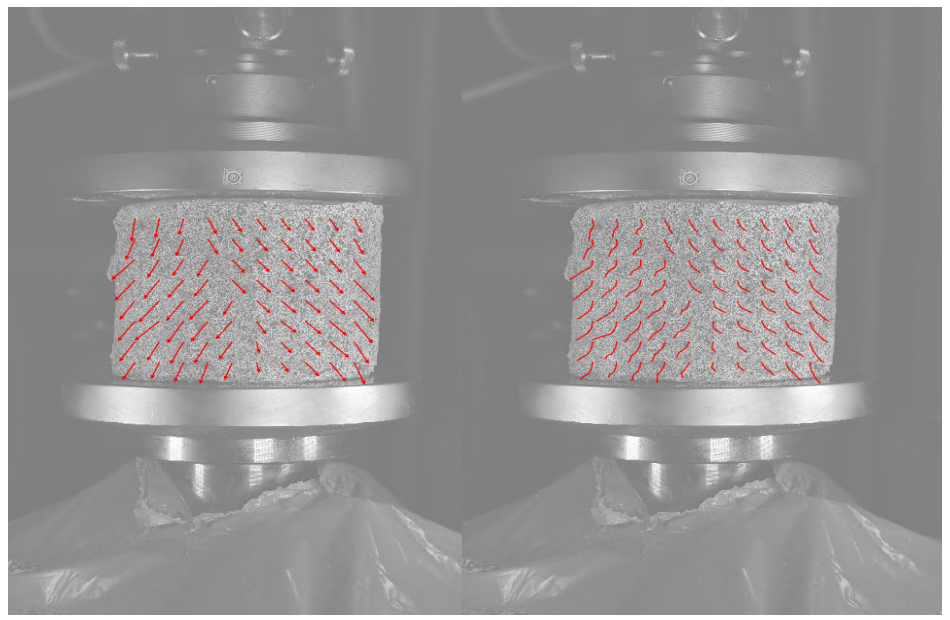

Fig. 4 The deformation vectors of the mesh grid (left), and the paths of the mesh points (right) put on the image of the soil sample.

Fig. 3 (right) presents the virtual mesh applied to the sample in order to obtain the real pathways of the mesh-points, chosen on the surface of the soil sample. The deformation vectors of the mesh grid (left) and the paths of the mesh points (right) put on the image of the soil sample are presented in Fig. 4. From that figure, one may observe the shear failure 
behaviour of the sample. The values are resized in order to notice this. It is even more apparent when colours are applied to illustrate the strains.
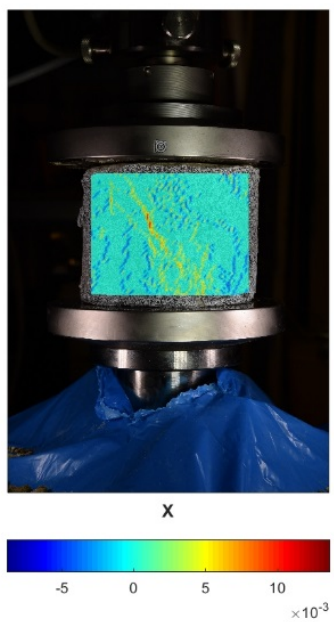
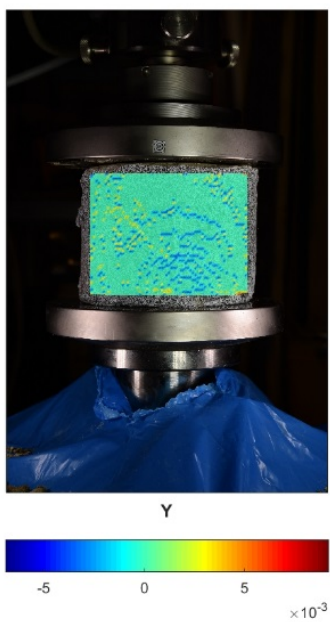

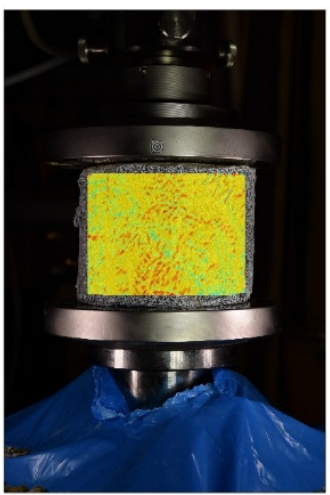

$\mathrm{XY}$

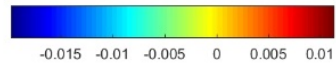

Fig. 5 The strain tensor components $\left(\varepsilon_{\mathrm{xx}}, \varepsilon_{\mathrm{yy}}, \varepsilon_{\mathrm{xy}}\right)$ digital maps at the early stage of the experiment, put on the image of the soil sample.

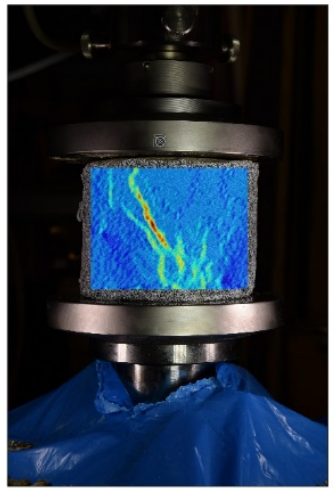

$\mathrm{x}$

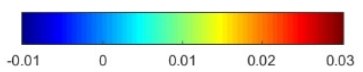

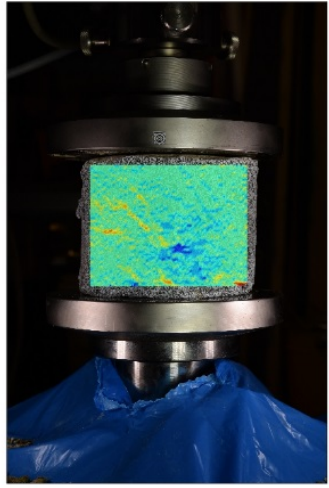

Y

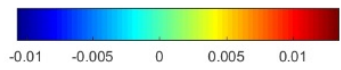

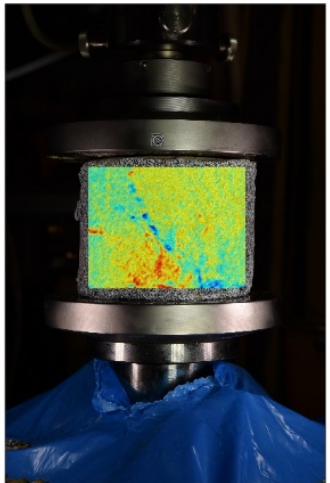

$\mathrm{XY}$

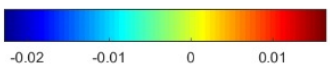

Fig. 6 The strain tensor components $\left(\varepsilon_{\mathrm{xx}}, \varepsilon_{\mathrm{yy}}, \varepsilon_{\mathrm{xy}}\right)$ digital maps during the medium phase of the experiment, put on the image of the soil sample.

Fig. 5 presents the digital maps of the strain tensor components, namely $\varepsilon_{x x}, \varepsilon_{y y}, \varepsilon_{x y}$, at the early stage of the experiment, put on the image of the soil sample. From the first tensor component $\varepsilon_{\mathrm{xx}}$ map, one may notice the beginning of the oblique crack, which is not visible in the other strain components, where the behaviour is chaotic. With an increase in force, the oblique crack is also visible in the tensor component $\varepsilon_{\mathrm{xy}}$ map, seen in Fig. 6. At the final phase of this experiment, another oblique crack is visible in Fig. 7, where the tensor component $\varepsilon_{\mathrm{yy}}$ map shows it, as well. 


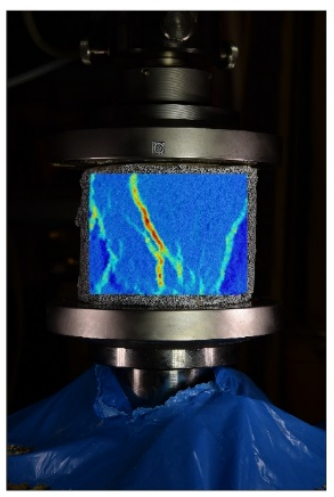

$\mathrm{X}$

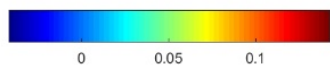

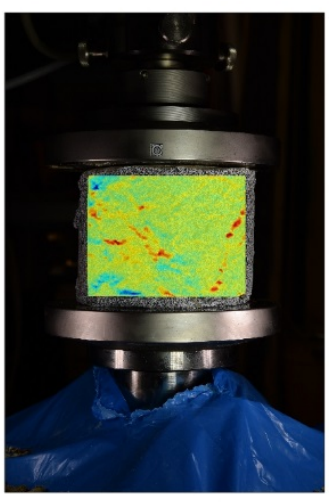

Y

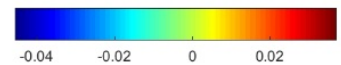

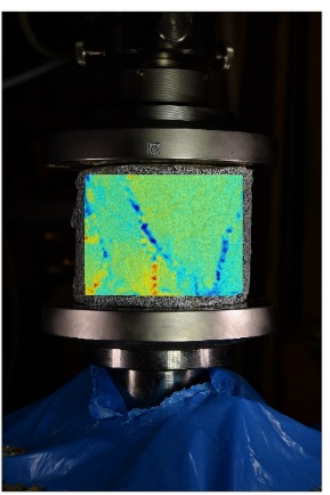

$X Y$

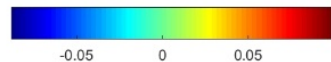

Fig. 7 The strain tensor components $\left(\varepsilon_{\mathrm{xx}}, \varepsilon_{\mathrm{yy}}, \varepsilon_{\mathrm{xy}}\right)$ digital maps at the end of the experiment, put on the image of the soil sample.

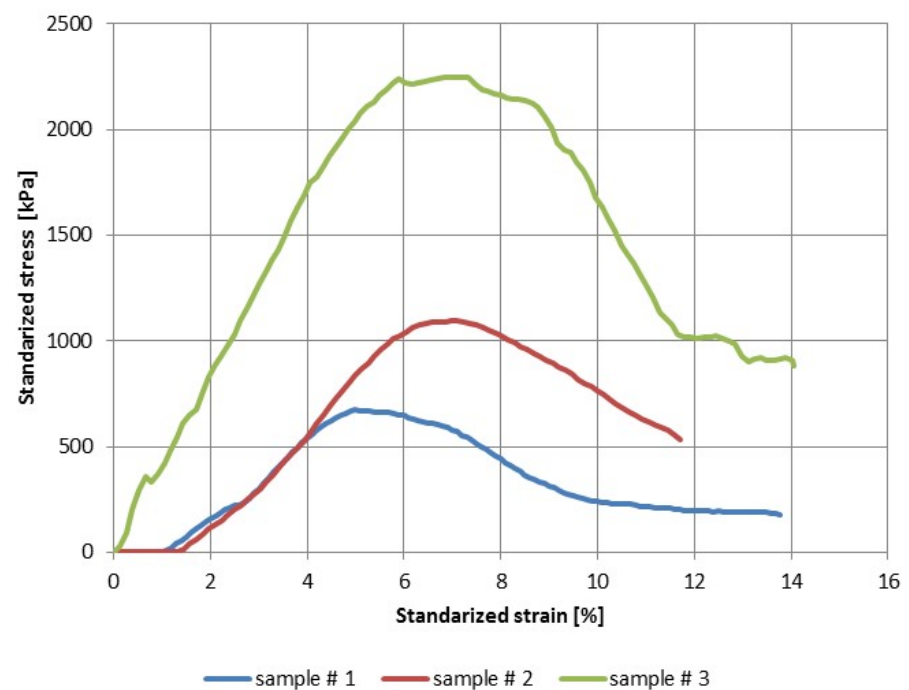

Fig. 8. Stress-strain curves, as received for the analysed soil samples.

Returning to Fig. 4, the first oblique crack is then visible after careful analysis of the vectors of the total deformations and the pathways of the mesh-points regularly chosen on the surface of the soil sample. Unfortunately, the second oblique crack is not visible in Fig. 4. As it was previously mentioned, the measurements of the compression loading and the vertical displacement has been performed during the test. Based on that data, Fig. 8 presents the stress-strain curves for all of the analysed samples. At the beginning of compression test for samples \#1 and \#2, the machine was adapting to the proper work, and thus, the stress is not visible, while displacements theoretically contributed more than $1 \%$ of strains. 
Table 1. Young modulus and Poisson ration of the analysed soil samples.

\begin{tabular}{|c|c|c|c|}
\hline Soil sample & $\begin{array}{c}\text { Young's } \\
\text { modulus } \boldsymbol{E} \\
{[\mathbf{M P a}]}\end{array}$ & $\begin{array}{c}\text { Poisson } \\
\text { Ratio } \\
\boldsymbol{v}[-]\end{array}$ & $\begin{array}{c}\text { Soil density } \\
\boldsymbol{\rho} \\
{\left[\mathbf{g} / \mathbf{c m}^{\mathbf{3}}\right]}\end{array}$ \\
\hline$\# 1$ & 23.6 & 0.26 & 2.18 \\
\hline$\# 2$ & 29.8 & 0.35 & 2.19 \\
\hline$\# 3$ & 43.4 & 0.34 & 2.17 \\
\hline
\end{tabular}

Table 1 presents the Young's modulus $E$ and Poison coefficient $v$ of the analysed samples in the range of stress-strains relation (ca. between $2 \%$ and $4 \%$ ), as well as the density $\rho$ of the soil samples.

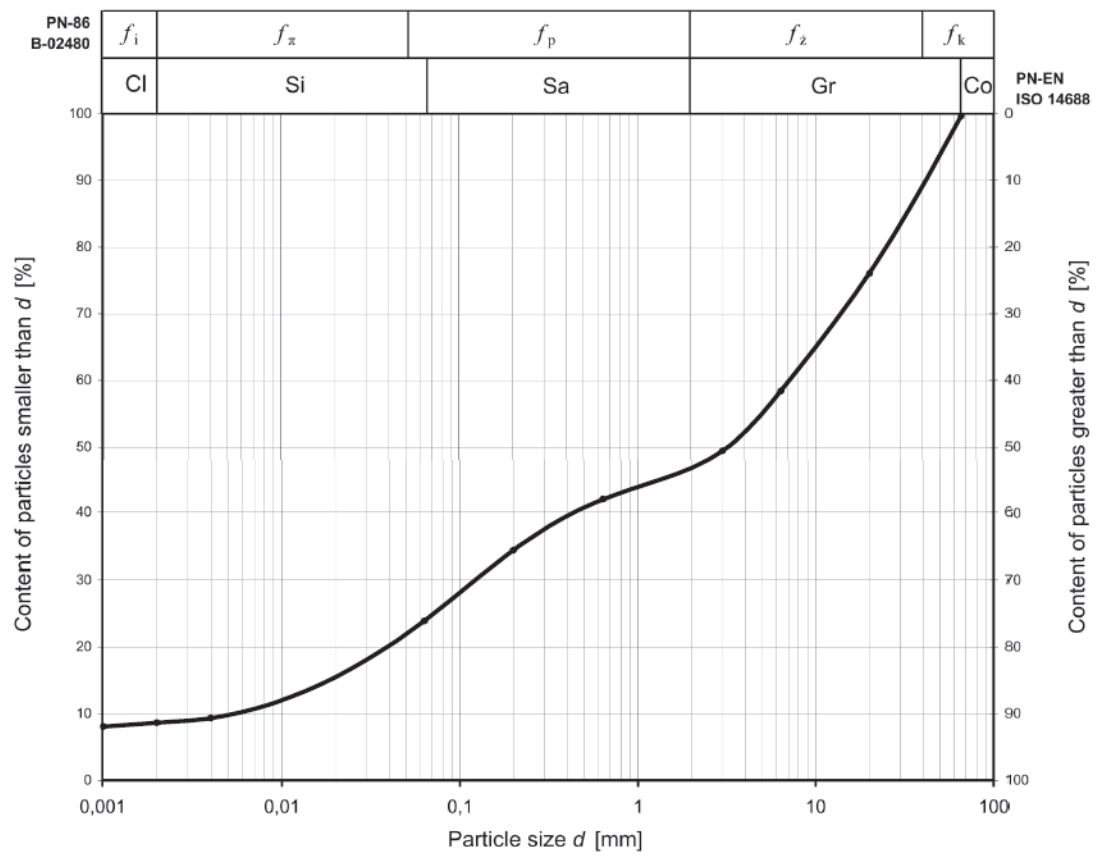

Fig. 9. Grain size distribution curve of the soil sample \# 3 .

The grain size distribution curve of the soil sample \#3 is visible in Fig. 9 and the data from Table 1 may be helpful in the identification of the soil. According to the EN ISO 14688 , the soil is classified as clayey gravel (clGr). However, bearing in mind the quality of that soil, the behaviour and the properties are those of cohesive clay rather than noncohesive gravel.

\section{Conclusions}

The paper presented the application of a non-contact optical method for investigating the deformation of a cohesive coarse soil material. For this type of soil, it is especially difficult to obtain basic strength parameters. Three soil samples were tested. The uniaxial compression tests of the soil samples performed indicate that the behaviour of the soil is 
more similar to cohesive clay rather than to non-cohesive gravel. The use of a non-contact optical method, followed by the study of a digital image correlation (DIC) of the sample obtained during tests effectively completes the description of the behaviour of this type of soil material.

\section{References}

1. M. Tekieli, S. De Santis, G. de Felice, A. Kwiecień, and F. Roscini (2017). Application of Digital Image Correlation to composite reinforcements testing, Composite Structures, V. 160, pp. 670-688

2. F. Lagattu, J. Brillaud, and M-C. Lafarie-Frent (2004), High strain gradient measurements by using digital image correlation technique, Materials Characterization 53, pp. 17-28

3. L. Chevalier, S. Calloch, F. Hild, and Y. Marco, Digital image correlation used to analyze the multiaxial behavior of rubber-like materials, Eur. J. Mech. A/Solids 20 (2001), pp. 169-187

4. T.C. Chu, W.F. Ranson, M.A. Sutton and W.H. Peters (1985), Applications of Digital.Image-Correlation Techniques to Experimental Mechanics, Experimental Mechanics, pp. 232-244

5. T. Majcherczyk, Z. Pilecki, Z. Niedbalski, E. Pilecka, M. Blajer, and J. Pszonka (2012). The influence of geological engineering and geotechnical conditions on parameter selection of the primary lining of a road tunnel in Laliki. Mineral Resources Management 28 (1), pp. 103-124

6. A. Kwiecień (2014) Shear bond of composites-to-brick applied with highly deformable, in relation to resin epoxy, interface materials. Mater Struct 47:2005-2020

7. A. Kwiecień (2015) Strengthening of masonry using natural fibers bonding with highly deformable adhesives. GSTF J Eng Technol 3(2)

8. M. Tekieli, M. Słoński (2013). Application of Monte Carlo filter for computer visionbased Bayesian updating of finite element model, Mechanics and Control, 32 (4), pp. 171-177

9. D. Łątka, M. Tekieli. Optical measurements in the field of masonry construction laboratory tests. $2^{\text {nd }}$ International Conference on Engineering Sciences and Technologies ESAT 2016, Tatranské Matliare, Slovak Republic 